\title{
Stability and Fidelity of the Finite Element Time Domain Method With Distorted Mesh
}

\author{
Boguslaw Butrylo, Christian Vollaire, and Laurent Nicolas
}

\begin{abstract}
Properties of the three-dimensional formulation of the finite element time domain algorithm for the wave equation are analyzed. First-order edge elements are implemented in the formulation. Several issues associated with deformation of the structured mesh and efficiency of the time integration scheme are presented. The convergence and stability of the time domain algorithm depending on the spatial discretization are discussed. The numerical accuracy of the simulation is studied.
\end{abstract}

Index Terms-Edge elements, finite element method, stability, time domain analysis.

\section{INTRODUCTION}

$\mathbf{T}$ HE finite element (FE) method is usefully applied in a wide variety of circumstances in computational electromagnetics. The fundamental advantage of this method is to create a smart numerical model with an unstructured and a structured grid. The FE scheme is more flexible in handling complicated geometry than other approximated numerical methods, but the creation of the suitable FE mesh is not a trivial task. Right physical sense of the edge elements compared to classical ones (nodal based) make this numerical method efficient to take into account discontinuities of the electromagnetic field. Moreover, time domain analysis is required for large number of applications. So, the vector FE time domain algorithm is useful in modeling of electromagnetic phenomena. The spatial discretization of an FE model and quality of the mesh have an effect on the properties of the time-domain algorithm (FETD) used for wave propagation problems. The value of the time step $\Delta t$ for a known FE grid must satisfy the Courant-Friedrichs-Levy (CFL) restriction [1]

$$
\Delta t \leq q \cdot \frac{\min (\Delta r)}{\nu}
$$

where $\Delta r$ is a dimension of an element, $\nu$ is the speed of an electromagnetic (EM) wave in a medium, and $q$ is the Courant number. The CFL condition is tightly coupled with the Nyquist's restriction

$$
\max (\Delta r) \leq 2 \cdot \frac{\nu}{f}
$$

Manuscript received July 1, 2003. This work was supported in part by the Development and Scientific Fund of Region Rhone-Alpes, France, under Grant 9610 6550, and in part by Project BTU W/WE/2/03.

B. Butrylo is with the Department of Theoretical Electrotechnics and Metrology, Bialystok Technical University, 15-950 Bialystok, Poland (e-mail: butrylo@vela.bialystok.pl)

C. Vollaire and L. Nicolas are with the Centre de Genie Electrique de Lyon (CEGELY), UMR CNRS 5005, Ecole Centrale de Lyon, 69134 Ecully, France (e-mail: Christian.Vollaire@ec-lyon.fr; Laurent.Nicolas@ec-lyon.fr).

Digital Object Identifier 10.1109/TMAG.2004.825426 where $f$ is the frequency of the propagating EM wave.

If the FE mesh is distorted or locally refined, the value of the Courant number depends on the quality of the mesh and the method of integration in the time domain. There is no clear rule to determine the optimal value of the $q$ coefficient. In this paper, the properties of the first-order edge-based FE grid is discussed. The objective of the paper is to analyze the stability and convergence of the FETD-edge element algorithm in order to estimate the minimum value of the Courant number.

\section{Finite ELEMENT SCHEME}

The mathematical background of the finite-element time-domain formulation is well known [1], [2]. The governing vector wave equation is derived from the Maxwell equations for some linear and isotropic media

$$
\nabla \times \frac{1}{\mu} \nabla \times \mathbf{e}+\sigma \frac{\partial \mathbf{e}}{\partial t}+\varepsilon \frac{\partial^{2} \mathbf{e}}{\partial t^{2}}=0 .
$$

Application of the Galerkin method and spatial discretization of the analyzed model yield to a matrix linear ordinary differential equation (ODE)

$$
\mathbf{T} \frac{d^{2} \mathbf{e}}{d t^{2}}+\mathbf{R} \frac{d \mathbf{e}}{d t}+\mathbf{S} \cdot \mathbf{e}+\mathbf{f}(t)=0
$$

The components of a mass matrix $\mathbf{T}$, a stiffness matrix $\mathbf{S}$, and a damping matrix $\mathbf{R}$ are stated by the following equations:

$$
\begin{aligned}
t_{i j} & =\int_{V_{p}} \varepsilon \mathbf{W}_{i} \mathbf{W}_{j} d V \\
s_{i j} & =\int_{V_{p}} \frac{1}{\mu}\left(\nabla \times \mathbf{W}_{i}\right)\left(\nabla \times \mathbf{W}_{j}\right) d V \\
r_{i j} & =\int_{V_{p}} \sigma \mathbf{W}_{i} \mathbf{W}_{j} d V+\int_{S_{A B C, p}} \mathbf{W}_{j} \frac{1}{\mu c}\left(\mathbf{W}_{i} \times \overrightarrow{\mathbf{n}}\right) d S .
\end{aligned}
$$

The $S_{A B C, p}$ indicates the external surface of the $p$ th element with predefined absorbing boundary conditions. The first-order Engquist-Majda boundary condition is implemented on the external surface of the FE model to modelize the unbounded domain [3]. The $\mathbf{f}(t)$ column vector represents the dynamic load in the analyzed model.

Since the numerical model is approximated by edge elements, $\mathbf{W}_{i}$ and $\mathbf{W}_{j}$ are the edge-based vector shape functions. In the elaborated algorithm, the first-order $\mathbf{H}(\operatorname{rot}, \Omega)$ tetrahedral elements are implemented [4], [5]. 
The edge element formulation is an alternative to the common, widely used nodal-base FE formalism. There are many circumstances in which edge elements have clear advantages over the typical nodal formulation [6]. The edge elements enforce tangential continuity of the electric (or magnetic) field on the edge of elements, while the normal field components are free. In this way, the implementation of the essential boundary conditions in the edge formulation is much easier than in nodal formulation, and the edge elements do not generate conflict between different types of boundary conditions.

Flexibility of the FE technique and right physical sense of the edge elements make this formulation useful in numerical modeling of electromagnetic phenomena. Of course, the edge-based formulation of the FE method is not perfect. The edge elements are not suitable when the sources of the electromagnetic field are not free of divergence [6].

The second, significant fault of the edge element formulation is the numerical efficiency. The edge elements are less efficient than nodal elements. The FE method based on edge elements needs more unknowns to obtain the same accuracy as the nodal formulation. Denoting by $N_{N}$ and $N_{E}$ the number of nodes and the number of edges in the model with structured FE grid, the approximate relation between these two quantities is represented as follows:

$$
N_{E} \cong 5.7 \cdot N_{N}
$$

The total number of degrees of freedom in the edge formulation is equal to the number of edges $\left(N_{E}\right)$, while in the nodal formulation the vector field is described by three components $\left(3 N_{N}\right)$. Nevertheless, the edge-based form of the FE model is more memory consuming than the equivalent nodal model. Equation (6) indicates larger computational cost of the edge formulation, since the total elapsed time of the solver increases when the number of degrees of freedom is higher. The nodal-based FE algorithm is less memory and computationally demanding.

\section{TIME DOMAIN MODELING}

The ordinary differential equation (4) is transferred into linear algebraic equation by discretization of the time domain. Two unconditionally stable (backward Euler scheme and Newmark method [1]) and two conditionally stable (central Euler and mixed Euler) time integration schemes are used in the algorithm. These time integration algorithms differ primary in the manner in which the first and second derivatives of field distribution are calculated. For example, the differential form of the ODE for the central Euler scheme is stated by the equation

$$
\left(\mathbf{T}+\frac{\Delta t}{2} \mathbf{R}\right) \cdot \mathbf{e}_{n+1}=\left(2 \mathbf{T}-\Delta t^{2} \mathbf{S}\right) \cdot \mathbf{e}_{n}+\left(\frac{\Delta t}{2} \mathbf{R}-\mathbf{T}\right) \cdot \mathbf{e}_{n-1}-\Delta t^{2} \mathbf{f}_{n}
$$

while the mixed time integration scheme results in the following form:

$\mathbf{T} \cdot \mathbf{e}_{n+1}=\left(2 \mathbf{T}-\Delta t \mathbf{R}-\Delta t^{2} \mathbf{S}\right) \cdot \mathbf{e}_{n}+(\Delta t \mathbf{R}-\mathbf{T}) \cdot \mathbf{e}_{n-1}-\Delta t^{2} \mathbf{f}_{n}$
The mixed time integration scheme is a combination of two basic methods of numerical derivation. The backward Euler scheme is implemented for the first derivative, while the second-order derivative in the time domain is approximated by the central Euler rule.

The backward scheme and Newmark method are unconditionally stable, but convergence of these methods depends on the time step $\Delta t$. When the backward Euler scheme is implemented to the ODE, the time-dependent distribution of electric field is described by the formula

$$
\left(\mathbf{T}+\Delta t \mathbf{R}+\Delta t^{2} \mathbf{S}\right) \cdot \mathbf{e}_{n}=(2 \mathbf{T}+\Delta t \mathbf{R}) \cdot \mathbf{e}_{n-1}-\mathbf{T} \cdot \mathbf{e}_{n-2}-\Delta t^{2} \mathbf{f}_{n}
$$

All implemented time integration schemes belong to a group of multistep methods, and the final, common form of the time domain matrix equations is [2]

$$
\mathbf{A} \cdot \mathbf{e}_{n}=\mathbf{b}_{n} \text {. }
$$

The A matrix is not diagonal, thus making the problem implicit. The form and properties of the $\mathbf{A}$ matrix arise from the implemented time integration scheme.

The properties of the time integration scheme have an effect on the accuracy, numerical stability, and convergence of the FETD algorithm. The central difference and mixed difference schemes are useful, if an explicit method of matrix solution is formulated. Unfortunately, the final matrix equation has to fulfill some additional restrictions in this case. The stability of conditionally stable schemes depends on the time integration step $\Delta t$. The backward scheme and Newmark method are unconditionally stable, but convergence of these methods depends on the time step $\Delta t$.

The preconditioned conjugate gradient algorithm (PCG) with the symmetric successive over relaxation (SSOR) preconditioner is applied to solve the final matrix equation [7]. The computational cost of the PCG algorithm is known to be approximately $N_{\mathrm{DOF}}^{1.5}$, but the quality of the mesh has an effect on the total cost of the iterative solver.

\section{NUMERICAL EXPERIMENTS}

The presented time domain algorithm is used to calculate a benchmark problem with a monochromatic plane wave propagating in free space. Several three-dimensional (3-D) rectangular domains are meshed with the same number of elements (46305 tetrahedral elements, 20 nodes per wavelength) leading to several distorted meshes.

Two types of structured tetrahedral grids are used in the simulation. The final FE grid is based on a preliminary hexahedral discretizaton of the 3-D rectangular model. The secondary grid is inscribed into the hexahedral mesh and consists of tetrahedral edge elements. In this approach, the primary (hexahedral) mesh is the same for both types of the final grid. The properties of the FE mesh become different in the second step of the model discretization, because other methods of transition from hexahedra to tetrahedral elements are used (Fig. 1). The final properties of the whole FE mesh arise from these elementary schemes of domain decomposition. 


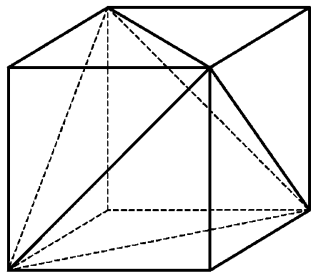

(a)

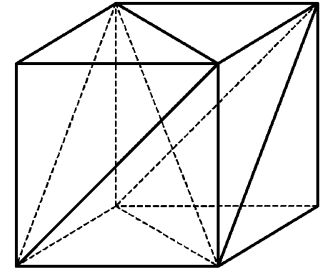

(b)
Fig. 1. Two types of tetrahedral grid inscribed into a single cubic element: (a) mesh A; (b) mesh B.

TABLE I

PROPERTIES OF THE STRUCTURED TETRAHEDRAL MESH

\begin{tabular}{lcc}
\hline \hline \multicolumn{1}{c}{ Property } & Type A & Type B \\
\hline $\begin{array}{l}\text { Number of tetrahedral elements inscribed } \\
\text { into a single hexahedral element }\end{array}$ & 5 & 6 \\
\hline $\begin{array}{l}\text { Minimal value of } Q_{R} \text { coefficient if the } \\
\text { primary mesh is based on the cube }\end{array}$ & 0.794 & 0.794 \\
\hline $\begin{array}{l}\text { Maximal value of } Q_{R} \text { coefficient if the } \\
\text { primary mesh is based on the cube }\end{array}$ & 1.0 & 0.794 \\
\hline \hline
\end{tabular}

The unequivocal information about distortion of the tetrahedral mesh is expressed by the Robert/Roux factor [8]

$$
Q_{R}=N \cdot\left(\frac{V_{T}}{V_{S}}\right)^{\frac{1}{3}}
$$

where $N$ is a normalization coefficient, $V_{T}$ is the volume of a tetrahedron, and $V_{S}$ is the volume of a circumscribed sphere. This factor can change from 0 ( 4 points lie in a plane) to 1 (ideal, regular tetrahedral element).

The properties of these tetrahedral meshes are comparable only by the minimum value of the $Q_{R}$ coefficient (Table I).

Mesh B consists of topologically equivalent tetrahedral elements, because all of elements have the same value of the Robert/Roux coefficient. The optimal value of $Q_{R}$ factor $\left(Q_{R}=0.794\right)$ is obtained when this type of the mesh is inscribed into cubes. When the primary (hexahedral) mesh is distorted, the value of the Robert/Roux factor remains uniform but its value is less than the optimal one.

Mesh A is better shaped, however, two subsets of the tetrahedral elements can be distinguished in this matter. The first one consists of tetrahedral elements with better shaped coefficient $\left(Q_{R}=1\right.$ if the primary mesh is based on the cube). Only $20 \%$ of the elements have better $Q_{R}$ factor and the remainder is worse shaped. These elements have the same topological quality as the presented mesh B.

Some numerical computations enable us to determine a correlation between the quality of the FE tetrahedral mesh and the properties of the implemented time integration schemes. It is shown from the numerical results that the value of the Courant number must be decreased when the distortion of the FE mesh increases (Fig. 2). If the value of $q$ factor is greater than the value presented in the figure, the central and mixed Euler schemes are not stable.

It is also shown that the difference between meshes A and B for the central Euler scheme is perceptible only when the ideal FE mesh is created. If the value of the $Q_{R}$ factor is less than 0.7, the maximum $q$ coefficient is equal for these types of tetrahedral

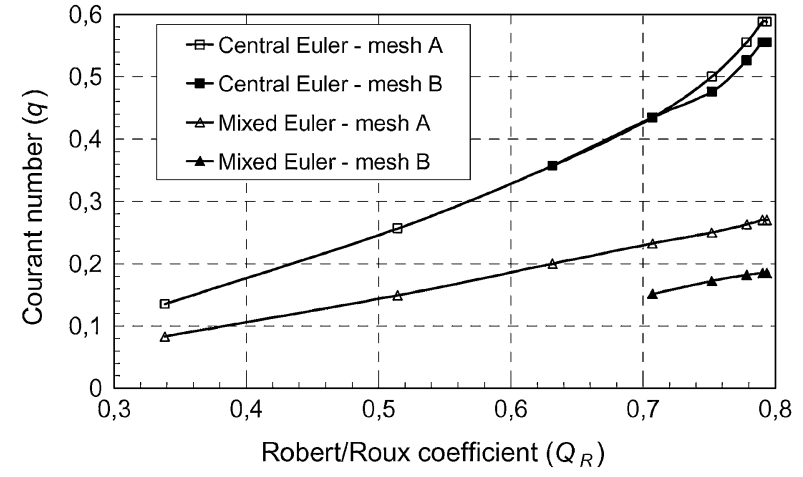

Fig. 2. Maximal value of $q$ factor as a function of mesh deformation.

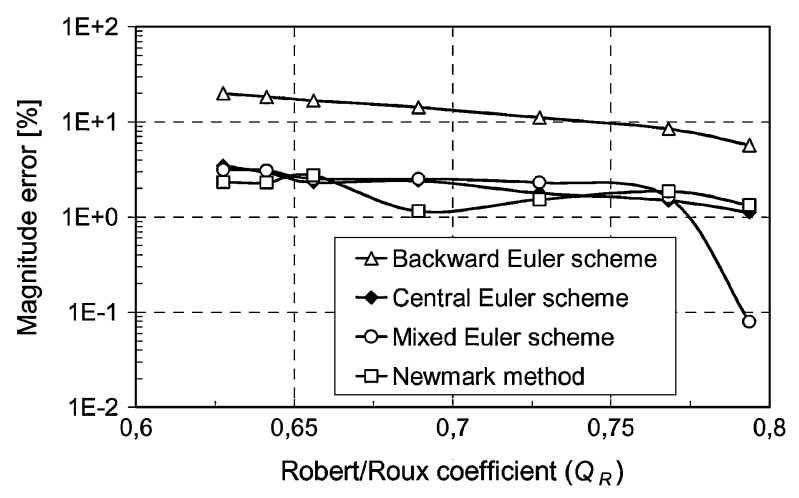

Fig. 3. Maximum value of magnitude error for uniform, distorted mesh.

grid. Both conditionally stable schemes do not converge when the inferior mesh (B) is significantly distorted $\left(Q_{R}<0.615\right.$ for the central Euler scheme, and $Q_{R}<0.7$ for the mixed scheme). The set of tetrahedral elements with better shape characteristic improves the overall stability of the time domain algorithm for mesh A. This essential factor of stabilization is lost for mesh B.

The mixed time integration scheme needs a lower $q$ coefficient than the central scheme. This means that the value of time step $\Delta t$ must be smaller, and the computational cost of the FETD algorithm increases. However, the mixed scheme is useful in explicit formulation of the algorithm, because the mass matrix has to be lumped solely.

The numerical results obtained from the time domain method are verified and compared to the analytical solution. The backward Euler method introduces the largest magnitude error (Fig. 3). Its maximal value is about $20 \%$. The fidelity of the Newmark method is better than the backward scheme for both the ideal and distorted meshes. The maximum magnitude error for conditionally stable schemes is less than 5\%. The value of the error for these schemes increases two times when the distortion is changed in a narrow scope $Q_{R} \in\langle 0.615,0.8\rangle$.

The good agreement between computational and analytical results demands the increase of the number of iterations in the PCG algorithm (Fig. 4). In this way, the efficiency of the PCG algorithm decreases, because more floating point operations in the PCG algorithm are required to find the final solution. When the distorted FE mesh is used, the matrix $\mathbf{A}$ becomes worse conditioned and nondiagonally dominant. The components on the diagonal are constant while some nondiagonal components are equal to them. The second part of the off-diagonal terms 


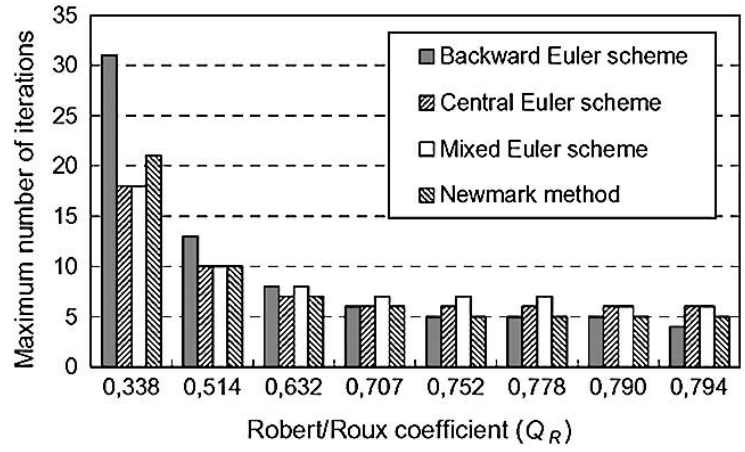

Fig. 4. Maximal number of iterations in PCG algorithm as a function of $Q_{R}$.

of $\mathbf{A}$ matrix goes to zero when the distortion increases. The entries of the A matrix for conditionally stable time integration schemes are the result of the dot product of the vector shape functions $\mathbf{W}_{i}$ and $\mathbf{W}_{j}$. The vector product is included to the terms of the A matrix when the unconditionally stable schemes are implemented. The behavior of the individual components of the A matrix depends on the orientation of the appropriate edges and the deformation of the FE mesh.

\section{CONCLUSION}

The stability and accuracy of the FETD method depend on the value of space increment $\Delta r$ and the value of time increment $\Delta t$. The value of the Courant number depends on the local distortion and the whole statistic of the Robert/Roux factor in the analyzed FE model.

The presented results indicate the tight relation between the performance of the algorithm and the quality of the FE mesh. The results concern two types of structured FE meshes with the first-order tetrahedral edge elements. The stability and accuracy of the algorithm with unstructured mesh depend on the local quality of the grid and the total statistic of the $Q_{R}$ coefficient in the model. The distorted FE edge-based mesh makes accuracy of conditionally and unconditionally schemes worse. Local refinements and distortions of the edge-based mesh cause global instability of the time domain scheme.

The conditionally stable schemes require fewer iterations of the PCG algorithm but the time step must be adapted to the quality of mesh. This means that the computational cost of the FETD algorithm depends on the quality of the edge-based mesh. Flexible changes of the time step enable us to modify the computational cost of the unconditionally stable schemes.

A well-shaped and fine FE mesh leads to a well-conditioned formulation of the FE time domain algorithm. In this case, the total computational cost and/or size of the FETD model can exceed the computational power of the modern computer hardware. The concurrent modeling of FE phenomena is an alternative approach to this problem.

\section{REFERENCES}

[1] U. Navsariwala and S. Gedney, "An unconditionally stable parallel finite element time domain algorithm," presented at the Joint IEEE Antennas and Propagation Society/United Radio Science Institute (APS/URSI) Symp., Baltimore, MD, July 1996.

[2] J.-F. Lee, R. Lee, and A. Cangellaris, "Time-domain finite-element methods," IEEE Trans. Antennas Propagat., vol. 45, pp. 430-441, Mar. 1997.

[3] B. Engquist and A. Majda, "Absorbing boundary conditions for numerical simulation of waves," Math. Computation, vol. 31, no. 139, pp. 629-651, 1977.

[4] A. Ahagon, K. Fujiwara, and T. Nakata, "Comparison of various kinds of edge elements for electromagnetic field analysis," IEEE Trans. Magn., vol. 32, pp. 898-901, May 1996.

[5] A. Bossavit, "A rationale for 'edge-elements' in 3-D fields computations," IEEE Trans. Magn., vol. 24, pp. 74-79, Jan. 1988.

[6] G. Mur, "Edge elements, their advantages and their disadvantages," IEEE Trans. Magn., vol. 30, pp. 3552-3557, Sept. 1994.

[7] C. Vollaire and L. Nicolas, "Preconditioning techniques for the conjugate gradient solver on a parallel distributed memory computer," IEEE Trans. Magn., vol. 34, pp. 3347-3350, Sept. 1998.

[8] P. W. Daly. (1994) The Tetrahedron Quality Factors of CSDS. Max-Planck-Institut, Saarbrücken, Germany. [Online]. Available: http://www.mpae.gwdg.de/ daly/tetra.html 